The University of Southern Mississippi

The Aquila Digital Community

Faculty Publications

$9-1-2011$

\title{
The Method of Approximate Particular Solutions for Solving Elliptic Problems with Variable Coefficients
}

\author{
C.S. Chen \\ University of Southern Mississippi, CS.Chen@usm.edu \\ Chia-Ming Fan \\ National Taiwan Ocean University, cmfan@ntou.edu.tw \\ Pihua Wen \\ University of London, p.h.wen@qmul.ac.uk
}

Follow this and additional works at: https://aquila.usm.edu/fac_pubs

Part of the Physical Sciences and Mathematics Commons

\section{Recommended Citation}

Chen, C., Fan, C., Wen, P. (2011). The Method of Approximate Particular Solutions for Solving Elliptic Problems with Variable Coefficients. International Journal of Computational Methods, 8(3), 545-559. Available at: https://aquila.usm.edu/fac_pubs/353

This Article is brought to you for free and open access by The Aquila Digital Community. It has been accepted for inclusion in Faculty Publications by an authorized administrator of The Aquila Digital Community. For more information, please contact Joshua.Cromwell@usm.edu. 


\title{
The Method of Approximate Particular Solutions for Solving Elliptic Problems with Variable Coefficients
}

\author{
C.S. Chen, C.M. Fan ${ }^{\dagger}$ P.H. Wen ${ }^{\ddagger}$
}

\begin{abstract}
A new version of the method of approximate particular solutions using radial basis functions has been proposed for solving a general class of elliptic partial differential equations. In the solution process, the Laplacian is kept on the left-hand side as a main differential operator. The other terms are moved to the right-hand side and treated as part of the forcing term. In this way the close-form particular solution is easy to obtain using various radial basis functions. The numerical scheme of the new method of approximate particular solutions is simple to implement and yet very accurate. Three numerical examples are given and the results are compared to Kansa's method and the method of fundamental solutions.
\end{abstract}

Key words: Radial basis functions, particular solution, homogeneous solution, meshless method, elliptic partial differential equations.

\section{Introduction}

The particular solution has traditionally played an important role for solving ordinary and partial differential equations. The particular solution is known as the solution that satisfies the given differential equation in the infinite domain without the consideration of boundary conditions. In 1967 Fox, Henrici, and Moler [7] first proposed the method of particular solutions (MPS) to solve Laplace eigenvalue problem $\Delta u=-\lambda u$ in $\Omega$ and $u=0$ on $\partial \Omega$ where $\Omega$ is L-shape domain. The basic idea of the original MPS is to choose a set of basis functions $\phi_{i}(x, y)$ that satisfy the eigenvalue equation. We then try to find a solution of the following form

$$
u(x, y)=\sum_{i=1}^{\infty} a_{i} \phi_{i}(x, y)
$$

${ }^{*}$ Department of Mathematics, University of Southern Mississippi, Hattiesburg, MS 39406, USA.

${ }^{\dagger}$ Department of Harbor and River Engineering, National Taiwan Ocean University, Keelung 20224, Taiwan

${ }^{\ddagger}$ Department of Engineering, Queen Mary, University of London, London E1 4NS, UK

Correspondence to: C.S. Chen, E-mail: cschen.math@gmail.com 
which is also zero on the boundary $\partial \Omega$. In [7], the basis functions are the combination of Bessel functions and sine functions. The solution procedure of the MPS is similar to the method of fundamental solutions (MFS) [6, 8] which was proposed by Kupradze and Aleksidze [10] in 1964. In the MFS, the solution can be written as a linear combination of the fundamental solution which satisfies the given homogeneous equation. The fundamental solution is in fact a special type of particular solution. Similar to the MPS, we need only to impose the boundary condition for the MFS to solve the homogeneous equation. Both of the MPS and MFS were developed in the 1960's but later received less attention in the science and engineering communities. One of the reasons for these two methods fading away is that both methods are restricted for solving very specific type of problems. In contrast, during the same period of time the finite element method and finite difference method became very popular due to their ability to solve a large class of partial differential equations. The aim of this paper is to develop a meshless method using the concept similar to the MPS through the use of the radial basis functions to solve general types of partial differential equations. To distinguish our approach of using approximate particular solution from the original MPS in [7], we call our proposed numerical scheme as the method of approximate particular solutions (MAPS).

In general, the analytic particular solution is only available for a very limited class of differential equations with forcing term. As a result, numerical schemes are required for obtaining the approximate particular solutions. It is known that the particular solution for a given differential equation is not unique. In recent years a rich variety of numerical techniques have been developed for this purpose [1, 4, 5, 8, 12]. Among them, radial basis functions (RBFs) have been very popular due to their effectiveness and simplicity of implementation [8]. In the past, obtaining the particular solution has been just part of the solution procedure. To obtain the full solution of the original differential equation, the homogeneous solution is required. Recently the method of fundamental solutions (MFS) has coupled with the particular solution approach to form a one-stage approach to solve the partial differential equations with variable coefficients $[2,14]$. In the MFS, the fictitious boundary is required in the solution process. However, it is not trivial how to place the fictitious boundary so that the optimal numerical results can be obtained. The location of the source points on the fictitious boundary is still an outstand research problem. To alleviate this difficulty, a newly developed method of approximate particular solutions (MAPS) [3] is proposed without the use of the MFS. However, it can only be used to solve linear partial differential equations with the availability of close-form particular solution for the chosen RBF. Despite the recent advancement in obtaining the close-form particular solutions for various types of differential operators [4, 8, 13], we are still restricted to solve the linear partial differential equations. As shown in [2], the fundamental solution and the particular solution are put together as a one-stage approach. As a result, we are able to combine the basic concept of these two approaches to form a new MAPS that enables us to solve a general class of partial differential equations. This approach is somehow similar to the dual reciprocity boundary element method (DRBEM) [11] which is very popular in the area of boundary element methods. 
The structure of this paper is as follows. In Section 2, we provide the detail of the formulation of the proposed MAPS. In Section 3, we test our proposed method on three examples. We also make comparison of our approach with Kansa's method and the MFS. In Section 4, we draw the conclusion and give the potential applications of the MAPS.

\section{The Method of Approximate Particular Solutions}

The idea to extend the MAPS in [3] to more general types of equations which includes the variable coefficients is similar to the formulation of the DRBEM [11] where the Laplacian is kept on the left-hand side as the main differential operator and all the other terms containing reaction and convection terms are moved to right-hand side and become part of the forcing term. To avoid the domain integration, DRBEM is a very effective method for handling inhomogeneous problems and widely appears in the literature of boundary element methods. The success of the DRBEM depends on how the forcing term is approximated. RBFs have played an important role in this regard. In our proposed MPS, no domain or boundary integration is required. Extensive reviewed articles for numerical methods involving the particular solution have been given in the literature $[5,8]$. We refer readers to these papers for further details.

We consider the following elliptic partial differential equation in $2 \mathrm{D}$

$$
\begin{aligned}
\Delta u+\alpha(x, y) \frac{\partial u}{\partial x}+\beta(x, y) \frac{\partial u}{\partial y}+\gamma(x, y) u & =f(x, y), \quad(x, y) \in \Omega \\
\mathcal{B} u=g(x, y), \quad(x, y) & \in \partial \Omega
\end{aligned}
$$

where $\alpha(x, y), \beta(x, y), \gamma(x, y), f(x, y)$, and $g(x, y)$ are given functions. $\mathcal{B}$ is a boundary differential operator.

The key idea of the DRBEM and the new proposed MAPS is to rearrange (2) into Poisson-type equation; i.e.,

$$
\Delta u(x, y)=h\left(x, y, u, \frac{\partial u}{\partial x}, \frac{\partial u}{\partial y}\right), \quad(x, y) \in \Omega
$$

where

$$
h\left(x, y, u, \frac{\partial u}{\partial x}, \frac{\partial u}{\partial y}\right)=-\alpha(x, y) \frac{\partial u}{\partial x}-\beta(x, y) \frac{\partial u}{\partial y}-\gamma(x, y) u+f(x, y)
$$

Using RBFs, $\phi$, to approximate $h$, we have

$$
h\left(x, y, u, \frac{\partial u}{\partial x}, \frac{\partial u}{\partial y}\right)=\sum_{i=1}^{n} a_{i} \phi\left(r_{i}\right),
$$

where $r_{i}=\left\|(x, y)-\left(x_{i}, y_{i}\right)\right\|$ and $\left\{\left(x_{i}, y_{i}\right)\right\}_{1}^{n}$ are called the centers or trial points and $\phi: \mathbb{R}_{+} \rightarrow \mathbb{R}$ is a univariate function. 
Using RBF, an approximate particular solution to (2) is given by

$$
\hat{u}_{p}(\mathbf{x})=\sum_{i=1}^{n} a_{i} \Phi\left(r_{i}\right)
$$

where $\Phi$ is obtained by analytically solving

$$
\Delta \Phi=\phi .
$$

$\Phi$ in (8) can be obtained by repeated integration of $\phi[8]$. Three commonly used RBFs $\phi$ and their corresponding particular solutions $\Phi$ are shown in Table 1.

Table 1: Radial basis functions and their corresponding particular solutions.

\begin{tabular}{c|l}
\hline$\varphi$ & \multicolumn{1}{|c}{$\Phi$} \\
\hline$\sqrt{r^{2}+c^{2}}$ & $\frac{1}{9}\left(4 c^{2}+r^{2}\right) \sqrt{r^{2}+c^{2}}-\frac{c^{3}}{3} \ln \left(c+\sqrt{r^{2}+c^{2}}\right)$ \\
\hline$r^{2 m} \ln r$ & $\frac{r^{2 m+2} \ln r}{4(m+1)^{2}}-\frac{r^{2 m+2}}{4(m+1)^{3}}$ \\
\hline$r^{2 m-1}$ & $\frac{r^{2 m+1}}{(2 m+1)^{2}}$ \\
\hline
\end{tabular}

Let us assume the solution of (2) - (3) can be approximated by

$$
u(x, y) \simeq \hat{u}(x, y)=\sum_{i=1}^{n} a_{i} \Phi\left(r_{i}\right)
$$

Then from (8) we have

$$
\Delta u \simeq \Delta \hat{u}=\sum_{i=1}^{n} a_{i} \Delta \Phi\left(r_{i}\right)=\sum_{i=1}^{n} a_{i} \phi\left(r_{i}\right), \quad \text { in } \Omega .
$$

From (6) and (10), we have

$$
\sum_{i=1}^{n} a_{i} \phi\left(r_{i}\right)=-\alpha(x, y) \frac{\partial \hat{u}}{\partial x}-\beta(x, y) \frac{\partial \hat{u}}{\partial y}-\gamma(x, y) \hat{u}+f(x, y), \quad \text { for }(x, y) \in \Omega
$$

where

$$
\begin{aligned}
& \frac{\partial u}{\partial x} \simeq \frac{\partial \hat{u}}{\partial x}=\sum_{i=1}^{n} a_{i} \frac{\partial \Phi}{\partial x}\left(r_{i}\right) \\
& \frac{\partial u}{\partial y} \simeq \frac{\partial \hat{u}}{\partial y}=\sum_{i=1}^{n} a_{i} \frac{\partial \Phi}{\partial y}\left(r_{i}\right)
\end{aligned}
$$


(11) can be reformulated as follows:

$\sum_{i=1}^{n} a_{i}\left(\phi\left(r_{i}\right)+\alpha(x, y) \frac{\partial \Phi\left(r_{i}\right)}{\partial x}+\beta(x, y) \frac{\partial \Phi\left(r_{i}\right)}{\partial y}+\gamma(x, y) \Phi\left(r_{i}\right)\right)=f(x, y), \quad$ for $(x, y) \in \Omega$.

On the other hand, the boundary condition in (3) becomes

$$
\sum_{i=1}^{n} a_{i} \mathcal{B} \Phi\left(r_{i}\right)=g(x, y), \quad(x, y) \in \partial \Omega
$$

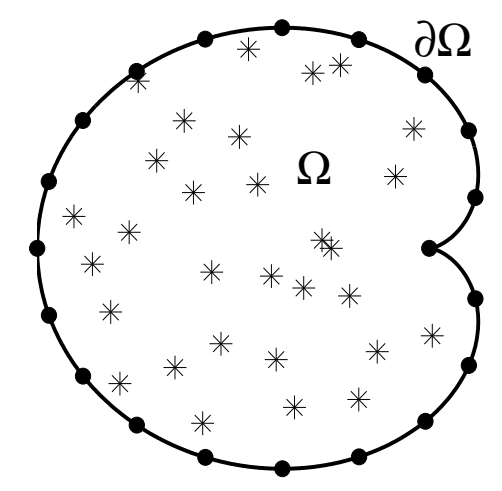

Figure 1: Interpolation points $(*)$, and boundary collocation points $(\bullet)$ in the computational domain.

For the numerical implementation, we need to choose two sets of interpolation points as shown in Figure 1. We let $n_{i}$ be the number of interior points $(*),\left\{\left(x_{j}, y_{j}\right)\right\}_{1}^{n_{i}}$, and $n_{b}$ the number of boundary points $(\bullet),\left\{\left(x_{j}, y_{j}\right)\right\}_{n_{i}+1}^{n_{i}+n_{b}}$. Furthermore, $n$ denotes the total number of points; i.e., $n=n_{i}+n_{b}$. By collocation method, from (14)-(15) we have

$$
\begin{aligned}
\sum_{i=1}^{n} a_{i} \Psi\left(r_{i j}\right) & =f\left(x_{j}, y_{j}\right), \quad 1 \leq j \leq n_{i}, \\
\sum_{i=1}^{n} a_{i} \mathcal{B} \Phi\left(r_{i j}\right) & =g\left(x_{j}, y_{j}\right), \quad n_{i}+1 \leq k \leq n,
\end{aligned}
$$

where

$$
\Psi\left(r_{i j}\right)=\phi\left(r_{i j}\right)+\alpha\left(x_{j}, y_{j}\right) \frac{\partial \Phi\left(r_{i j}\right)}{\partial x}+\beta\left(x_{j}, y_{j}\right) \frac{\partial \Phi\left(r_{i j}\right)}{\partial y}+\gamma\left(x_{j}, y_{j}\right) \Phi\left(r_{i j}\right)
$$


and $r_{i j}=\left\|\left(x_{j}, y_{j}\right)-\left(x_{i}, y_{i}\right)\right\|$. The above system of equations can be easily solved by standard matrix solver. Once $\left\{a_{i}\right\}_{1}^{n}$ is determined, the approximate particular solution in (9) becomes the approximate solution $\hat{u}$ of (2)-(3); i.e.,

$$
\hat{u}(x, y)=\sum_{i=1}^{n} a_{i} \Phi\left(r_{i}\right) .
$$

The above approach not only avoids the calculation of homogeneous solution but also extends the MAPS to solving partial differential equation with variable coefficients. Moreover, the corresponding particular solution of a given RBF can be obtained easily since the differential operator has been reduced to Laplacian as shown in (4). The solution procedure is fairly simple which is another advantage of our proposed method.

\section{$3 \quad$ Numerical Results}

To demonstrate the efficacy and consistency of the proposed MAPS, three numerical examples are considered in this section. We examined possible factors that will affect the accuracy, such as the number of nodes, the different type of RBF, the order of RBF, etc. The numerical results are compared with the analytical solution, Kansa's method [9], and the MFS $[2,8]$. In addition, we examine a practical guide for determining the optimal shape parameter of multiquadric (MQ), $c$, by tracking the residual with the known boundary conditions.

In order to evaluate the numerical errors, we adopted the root-mean-square error (RMSE) and the root-mean-square error of the derivative with respect to $x$ (RMSEx), which are defined as follows:

$$
\begin{aligned}
R M S E & =\sqrt{\frac{1}{n_{t}} \sum_{j=1}^{n_{t}}\left(\widehat{u}_{j}-u_{j}\right)^{2}}, \\
R M S E x & =\sqrt{\frac{1}{n_{t}} \sum_{j=1}^{n_{t}}\left(\frac{\partial \widehat{u}_{j}}{\partial x}-\frac{\partial u_{j}}{\partial x}\right)^{2}},
\end{aligned}
$$

where $n_{t}$ is the number of testing nodes chosen randomly in the domain. $\widehat{u}_{j}$ denotes the approximate solution at the $j^{t h}$ node. Note that we did not show the numerical results of RMSEy since they are very similar to RMSEx.

It is not trivial to determine the optimal shape parameter of MQ. In this paper we will follow the numerical scheme proposed in [3] to identify the optimal shape parameter by tracking the given boundary conditions. The definition of the residual of Dirichlet 
and Neumann boundary conditions are defined as follows:

$$
\begin{aligned}
& \text { ResidualD }=\frac{1}{n_{r}} \sum_{j=1}^{n_{r}}\left|\widehat{u}_{j}-g_{j}\right|, \\
& \text { ResidualN }=\frac{1}{n_{r}} \sum_{j=1}^{n_{r}}\left|\frac{\partial \widehat{u}_{j}}{\partial n}-g_{j}\right|,
\end{aligned}
$$

where $n_{r}$ is the number of test nodes on the boundary, $\partial \Omega$, and $g_{j}$ is the given boundary condition at the $j^{\text {th }}$ node.

Example 1 First, we consider the convection-diffusion-reaction equation with Dirichlet boundary condition as follows:

$$
\begin{aligned}
k \Delta u+\left(x^{2}+y^{2}\right) u+y \cos (y) \frac{\partial u}{\partial x}+\sinh (x) \frac{\partial u}{\partial y} & =f(x, y), \quad(x, y) \in \Omega, \\
u & =g(x, y), \quad(x, y) \in \partial \Omega,
\end{aligned}
$$

where $f(x, y)$ and $g(x, y)$ are given according to the following analytical solution:

$$
u(x, y)=\sin (\pi x) \cosh (y)-\cos (\pi x) \sinh (y) .
$$

The domain is defined by the following star shape parametric equation:

$$
\Omega=\left\{(x, y) \mid x=\left(1+\cos ^{2}(4 \theta)\right) \cos \theta, y=\left(1+\cos ^{2}(4 \theta)\right) \sin \theta, 0 \leq \theta<2 \pi\right\} .
$$

The computational domain and the profile of the solution in the extended domain are shown in Figure 2. Unless otherwise specified, the following data are adopted through all of the tests in this example: $n_{i}=212, n_{b}=100, k=1, n_{t}=193$ and $n_{r}=80$.

Let us first consider the MQ as the basis function in our proposed scheme. The optimal shape parameter, $c$, is determined by tracking the residual of the known boundary condition [3]. In Figure 3, the RMSE and ResidualD are obtained with respect to various shape parameter, $c$. The optimal shape parameter is chosen when the minimum ResidualD appears. The close resemblance between these two curves is readily seen. Since the boundary condition is already known, we can determine the optimal $c$ through ResidualD.

Based on the above scheme for choosing optimal shape parameter, Table 2 shows the results of several tests. We note that the optimal shape parameter changed systematically for MAPS as the number of nodes increases. In Kansa's method it is more difficult to determine the optimal shape parameter. In addition, the results of MAPS are compared with Kansa's method. Both methods produce good results for using the optimal shape parameter. The MAPS, however, outperforms Kansa's method in the evaluation of the derivative.

The optimal shape parameter, RMSE, and RMSEx for various $k$ are shown in Table 3. In general, the smaller $k$ is more difficult for the numerical simulation. In this table, 

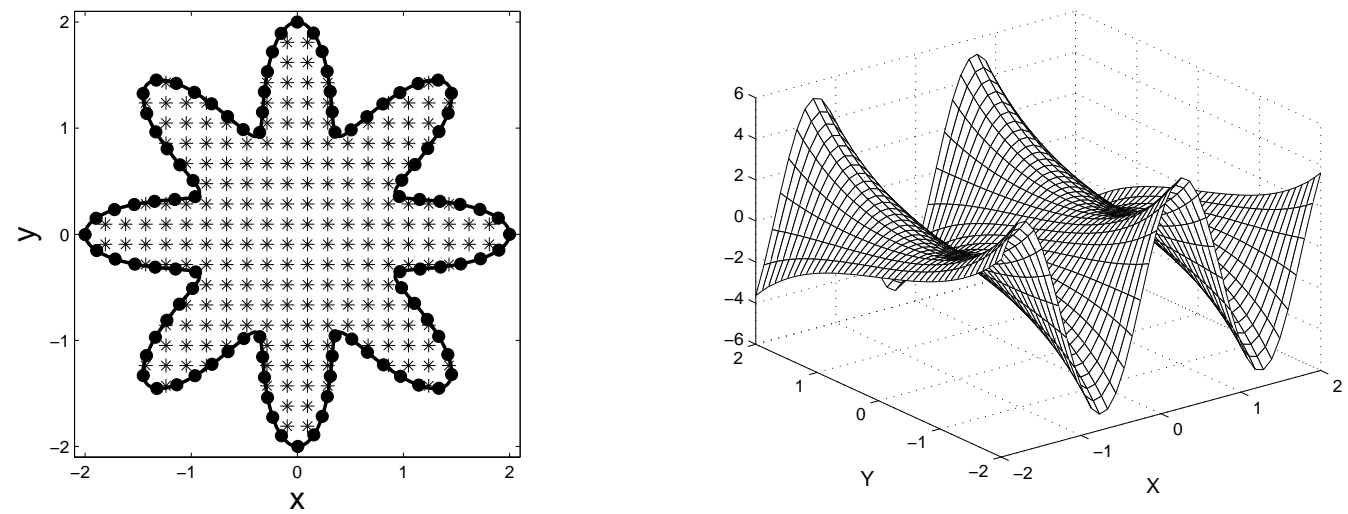

Figure 2: Computational domain (left) and profile of solution (right) in the extended domain.

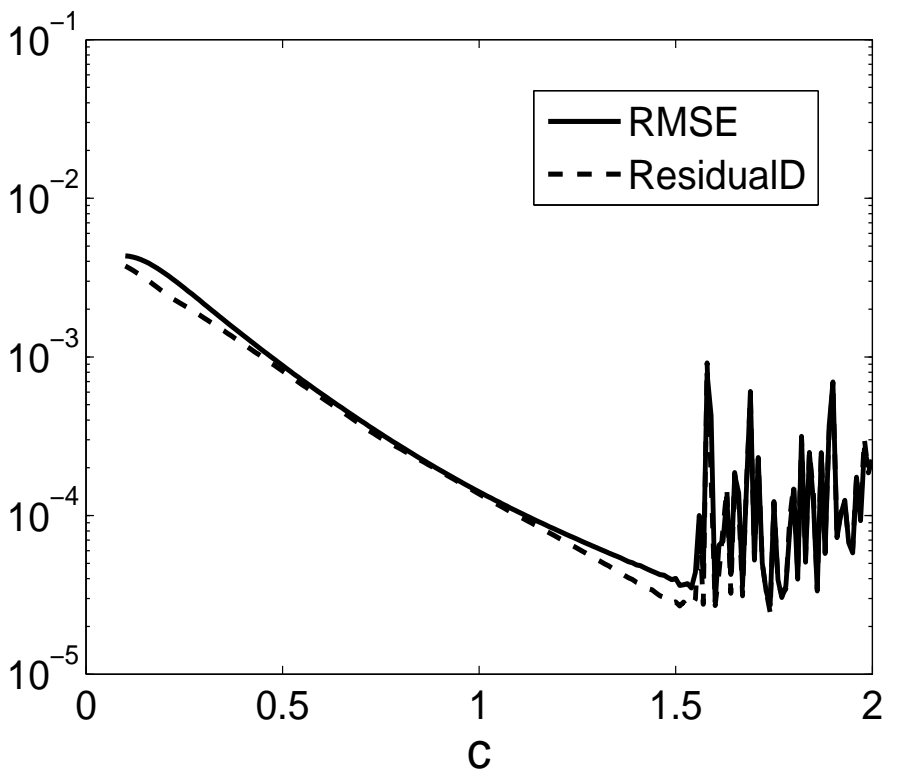

Figure 3: Profiles of RMSE and ResidualD with respect to different shape parameter, $c$.

the value of $k$ varies from 0.001 to 1,000 and little difference is observed. Hence the proposed meshless scheme is very stable for a large range of values of $k$.

To avoid the difficulty of choosing the optimal shape parameter, we choose the conical RBF, $r^{2 m-1}$, in the proposed scheme, and the results of different order of RBF are obtained as shown in Table 4. Three combinations of nodes are used to validate the consistency of the scheme and the higher-order RBF can acquire better solutions. Furthermore, the results are compared with the MFS in this table. The MAPS can produce comparable solution with the MFS. Also no fictitious source points are required. 
Table 2: Optimal shape parameter of MQ, RMSE and RMSEx obtained by different number of boundary nodes, $n_{b}$, and interpolation nodes, $n_{i}$.

\begin{tabular}{cccccc}
\hline \hline & & \multicolumn{2}{c}{ RMSE } & \multicolumn{2}{c}{ RMSEx } \\
\cline { 3 - 6 }$n_{i}$ & $n_{b}$ & MAPS & $\begin{array}{c}\text { Kansa's } \\
\text { method }\end{array}$ & MAPS & $\begin{array}{c}\text { Kansa's } \\
\text { method }\end{array}$ \\
\hline \multirow{2}{*}{113} & 50 & $6.24 E-4$ & $4.44 E-5$ & $8.70 E-3$ & $2.44 E-2$ \\
& & $(c=1.97)$ & $(c=0.03)$ & & \\
212 & 100 & $2.72 E-5$ & $1.33 E-6$ & $7.07 E-4$ & $2.88 E-3$ \\
& & $(c=1.74)$ & $(c=0.35)$ & & \\
317 & 150 & $8.95 E-6$ & $6.64 E-7$ & $9.98 E-5$ & $1.21 E-3$ \\
& & $(c=1.18)$ & $(c=0.33)$ & & \\
\hline \hline
\end{tabular}

Table 3: Optimal shape parameter of MQ, RMSE and RMSEx with respect to different $k$.

\begin{tabular}{cccc}
\hline \hline$k$ & $c$ & RMSE & RMSEx \\
\hline 0.001 & 1.68 & $6.32 E-5$ & $7.02 E-4$ \\
0.01 & 1.80 & $6.13 E-5$ & $1.02 E-3$ \\
0.1 & 1.62 & $4.43 E-5$ & $7.67 E-4$ \\
1 & 1.74 & $2.72 E-5$ & $7.07 E-4$ \\
10 & 1.77 & $3.12 E-5$ & $5.94 E-4$ \\
100 & 1.73 & $3.06 E-5$ & $9.04 E-4$ \\
1,000 & 1.65 & $2.80 E-5$ & $7.86 E-4$ \\
\hline \hline
\end{tabular}

Example 2 In the second example we consider the convection-diffusion equation as follows:

$$
\begin{aligned}
k \Delta u+\left(x^{2} y\right) u+\left(y^{2}+\cos x\right) \frac{\partial u}{\partial x}-y \sin x \frac{\partial u}{\partial y}=f(x, y), & (x, y) \in \Omega, \\
u=\sin \left(y^{2}+x\right)-\cos \left(y-x^{2}\right), & (x, y) \in \partial \Omega^{D}, \\
\frac{\partial u}{\partial n}=\left[\nabla\left(\sin \left(y^{2}+x\right)-\cos \left(y-x^{2}\right)\right)\right] \cdot \vec{n}, & (x, y) \in \partial \Omega^{N},
\end{aligned}
$$

where $\vec{n}$ is the unit normal vector and $f(x, y)$ depends upon the analytical solution, 
Table 4: RMSE and RMSEx obtained by different order of conical RBF, $r^{2 m-1}$.

\begin{tabular}{ccccccc}
\hline \hline & & & \multicolumn{2}{c}{ RMSE } & \multicolumn{2}{c}{ RMSEx } \\
\cline { 4 - 7 } & $n_{i}$ & $n_{b}$ & MAPS & MFS & MAPS & MFS \\
\hline$r^{7}$ & & & & & \\
& 113 & 50 & $4.63 E-3$ & $6.76 E-3$ & $4.29 E-2$ & $4.91 E-2$ \\
& 212 & 100 & $2.95 E-4$ & $2.32 E-4$ & $8.13 E-3$ & $4.05 E-3$ \\
& 317 & 150 & $6.52 E-5$ & $6.95 E-5$ & $9.11 E-4$ & $7.52 E-4$ \\
\hline$r^{9}$ & & & & & & \\
& 113 & 50 & $3.27 E-3$ & $4.25 E-3$ & $4.61 E-2$ & $3.11 E-2$ \\
& 212 & 100 & $1.31 E-4$ & $2.02 E-4$ & $3.21 E-3$ & $2.80 E-3$ \\
& 317 & 150 & $2.17 E-5$ & $3.36 E-5$ & $1.92 E-4$ & $4.24 E-4$ \\
\hline$r^{11}$ & & & & & & \\
& 113 & 50 & $2.24 E-3$ & $2.23 E-3$ & $2.90 E-3$ & $2.53 E-2$ \\
& 212 & 100 & $5.61 E-5$ & $4.72 E-4$ & $1.37 E-3$ & $3.69 E-3$ \\
& 317 & 150 & $4.97 E-5$ & $1.31 E-5$ & $6.56 E-4$ & $2.21 E-4$ \\
\hline \hline
\end{tabular}

which is given as follows:

$$
u=\sin \left(y^{2}+x\right)-\cos \left(y-x^{2}\right) .
$$

Let $\partial \Omega^{D}$ and $\partial \Omega^{N}$ be the boundaries subjected to Dirichlet and Neumann boundary conditions respectively and $\partial \Omega=\partial \Omega^{D} \cup \partial \Omega^{N}$. The portion of boundary above the xaxis has the Dirithlet boundary condition and the other portion is given the Neumann boundary condition.

The domain is defined by the following parametric equation:

$$
\Omega=\{(x, y) \mid x=\rho \cos \theta, y=\rho \sin \theta, 0 \leq \theta \leq 2 \pi\},
$$

where

$$
\rho=\left(\cos (2 \theta)+\sqrt{1.1-\sin ^{2}(2 \theta)}\right) .
$$

The computational domain and the profile of the solution in the extended domain are shown in Figure 4. The following data are used in all of the tests through this example: $n_{i}=101, n_{b}=40, k=1, n_{r}=200$, and $n_{t}=352$. We calculated the RMSE, Residual $N$ and ResidualD separately and plotted them in Figure 5. We observe that the profiles of these curves are similar. Therefore we can obtain the minimum $R M S E$ through the test of ResidualN and ResidualD on the boundary. 

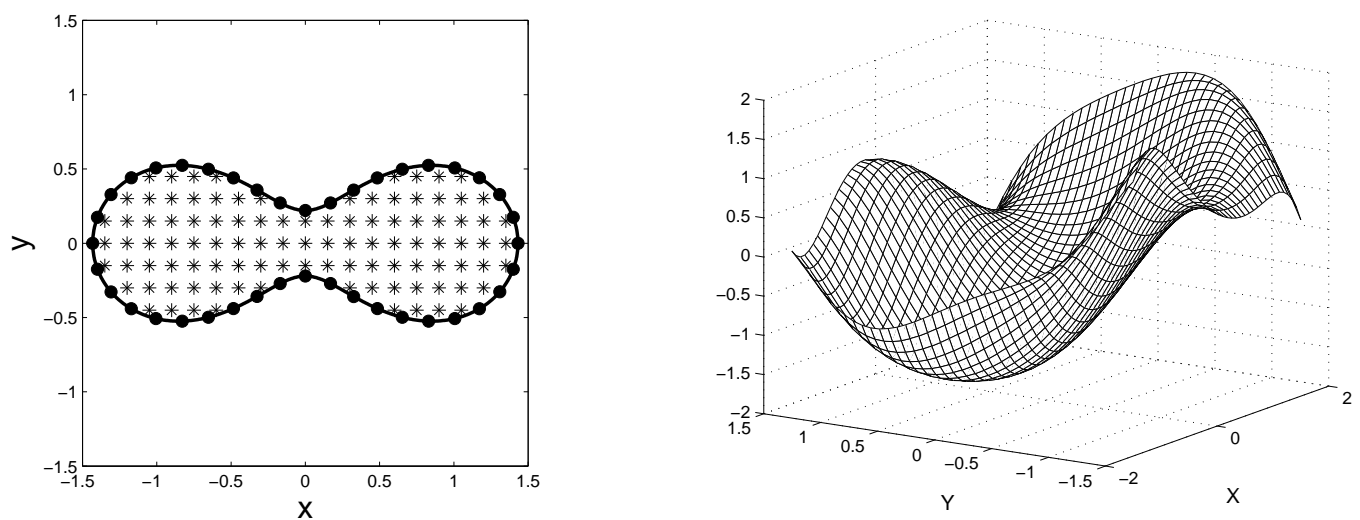

Figure 4: Computational domain (left) and profile of solution (right) in the extended domain.

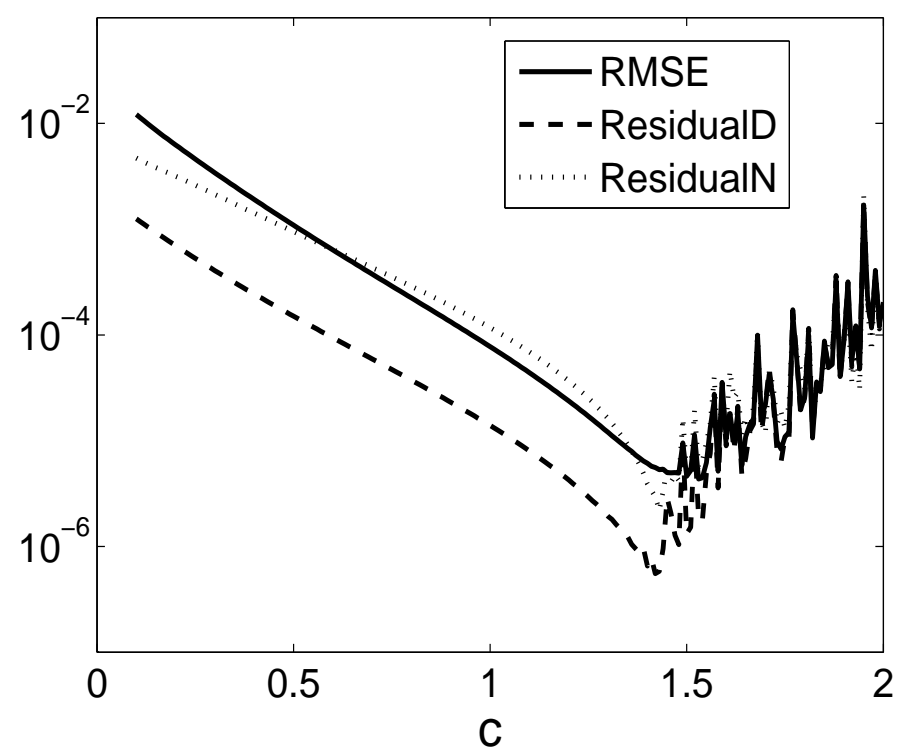

Figure 5: Profiles of RMSE, ResidualD and ResidualN with respect to different shape parameter, $c$.

The optimal shape parameter, RMSE, and $R M S E x$ obtained by using different numbers of nodes are given in Table 5 .

We also examine the performance of the scheme for different $k$. The results are given in Table 6. We observe that there is little difference in accuracy for various value of $k$.

Since we use the RBF for the Laplacian operator, we have many choices for the interpolating RBF. Here we use the conical RBF instead of MQ to avoid the difficulty of finding the optimal shape parameter. The RMSE and RMSEx obtained by different orders of RBF and different numbers of nodes are given in Tables 7 and 8. Increasing 
Table 5: Optimal shape parameter of MQ, RMSE and RMSEx obtained by different number of boundary nodes, $n_{b}$, and interpolation nodes, $n_{i}$.

\begin{tabular}{cccc}
\hline \hline$n_{i}$ & 52 & 101 & 145 \\
$n_{b}$ & 20 & 40 & 60 \\
\hline$c$ & 1.76 & 1.53 & 1.31 \\
RMSE & $2.33 E-4$ & $4.35 E-6$ & $3.51 E-6$ \\
RMSEx & $1.87 E-3$ & $1.98 E-5$ & $1.23 E-5$ \\
\hline \hline
\end{tabular}

Table 6: Optimal shape parameter of MQ, RMSE and RMSEx obtained by adopting different $k$.

\begin{tabular}{cccc}
\hline \hline$k$ & $c$ & RMSE & RMSEx \\
\hline 0.001 & 1.49 & $3.17 E-6$ & $1.65 E-5$ \\
0.01 & 1.36 & $1.52 E-6$ & $1.69 E-5$ \\
0.1 & 1.46 & $2.21 E-6$ & $1.48 E-5$ \\
1 & 1.53 & $4.35 E-6$ & $1.98 E-5$ \\
10 & 1.50 & $5.20 E-6$ & $1.90 E-5$ \\
100 & 1.50 & $4.76 E-6$ & $1.90 E-5$ \\
1,000 & 1.47 & $4.93 E-6$ & $1.82 E-5$ \\
\hline \hline
\end{tabular}

either the order of RBF or the numbers of nodes can have the effect of improving the accuracy.

Table 7: RMSE obtained by different order of conical RBF, $r^{2 m-1}$.

\begin{tabular}{cccccc}
\hline \hline$n_{i}$ & $n_{b}$ & $r^{5}$ & $r^{7}$ & $r^{9}$ & $r^{11}$ \\
\hline 101 & 40 & $8.35 E-4$ & $1.40 E-4$ & $2.71 E-4$ & $2.86 E-4$ \\
145 & 60 & $1.95 E-4$ & $6.39 E-5$ & $2.54 E-5$ & $2.10 E-5$ \\
202 & 80 & $2.29 E-4$ & $4.55 E-5$ & $4.22 E-6$ & $3.48 E-6$ \\
\hline \hline
\end{tabular}

Example 3 For the third example, we consider the following elliptic equation with vari- 
Table 8: RMSEx obtained by different order of conical RBF, $r^{2 m-1}$.

\begin{tabular}{cccccc}
\hline \hline$n_{i}$ & $n_{b}$ & $r^{5}$ & $r^{7}$ & $r^{9}$ & $r^{11}$ \\
\hline 101 & 40 & $1.63 E-3$ & $5.89 E-4$ & $8.29 E-4$ & $1.30 E-3$ \\
145 & 60 & $4.00 E-4$ & $1.44 E-4$ & $6.84 E-5$ & $9.73 E-5$ \\
202 & 80 & $3.92 E-4$ & $9.44 E-5$ & $1.57 E-5$ & $1.66 E-5$ \\
\hline \hline
\end{tabular}

able coefficients:

$$
\Delta u+\sin (x+y) u+\left(x y+x^{2}\right) \frac{\partial u}{\partial x}+x \sinh (y) \frac{\partial u}{\partial y}=f(x, y), \quad(x, y) \in \Omega,
$$

where $f(x, y)$ is given according to the following analytical solution:

$$
u=y \sin (\pi x)+x \cos (\pi y) .
$$

The computational domain and the profile of the solution in the square domain are shown in Figure 6. We use this example to show the convergence of the proposed method. We choose the conical RBF as the interpolating function instead of MQ so that no shape parameter is required. The boundary nodes and interior nodes are uniformly distributed in the domain and the minimum distance between two nodes are denoted as $d h$. $n_{t}$ is set equal to 500 .

Case I:

For the first test, we consider the Dirichlet boundary condition on the whole boundary of the square domain. The results of RMSE and RMSEx using different density of nodes are shown in Figure 7. The order of RBF, $m$, is set as 1, 2, and 3. From this figure we have results similar to those in the previous examples. Better accuracy can be obtained by either increasing the number of nodes or the order of RBF.

Case II:

Next, we consider the Neumann boundary condition along $y=0$. It is more challenging to solve such mixed-type boundary conditions than the previous case. The result of RMSE and RMSEx using different orders of RBF are shown in Figure 8. Overall, we observe the similar pattern in terms of convergence.

\section{Conclusions}

A two-stage numerical scheme using the MFS and the MAPS has been simplified. In the new approach, we do not require to find the homogeneous solution. The new proposed method uses the particular solution only to approximate the numerical solution of the given differential equation. Coupled with the idea from DRBEM, the MAPS is further 

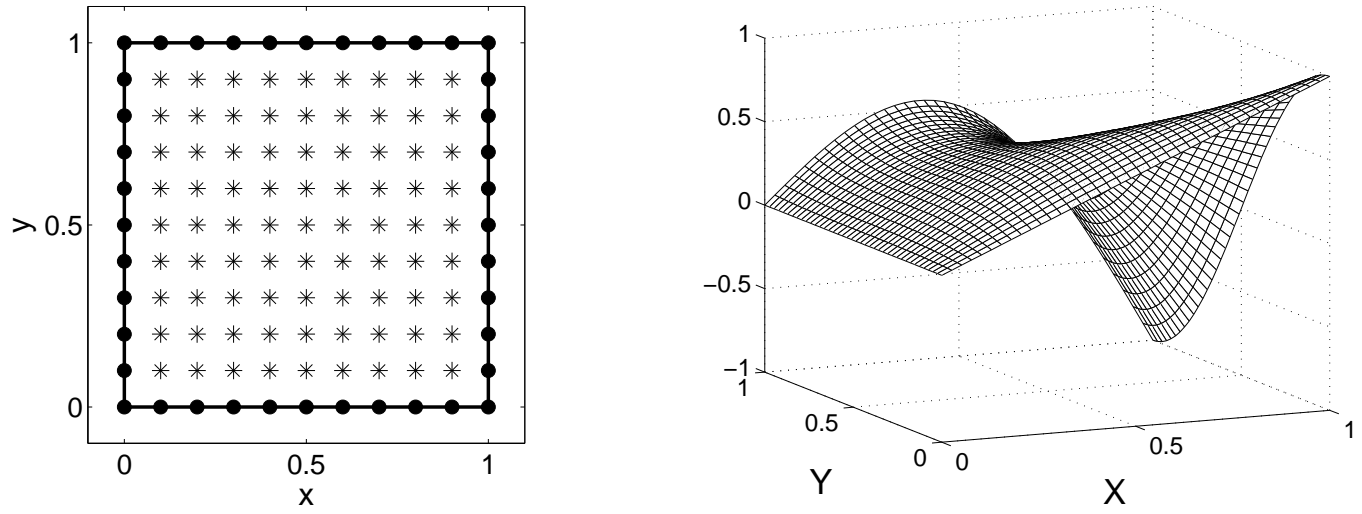

Figure 6: Computational domain (left) and profile of solution (right) in the square do-
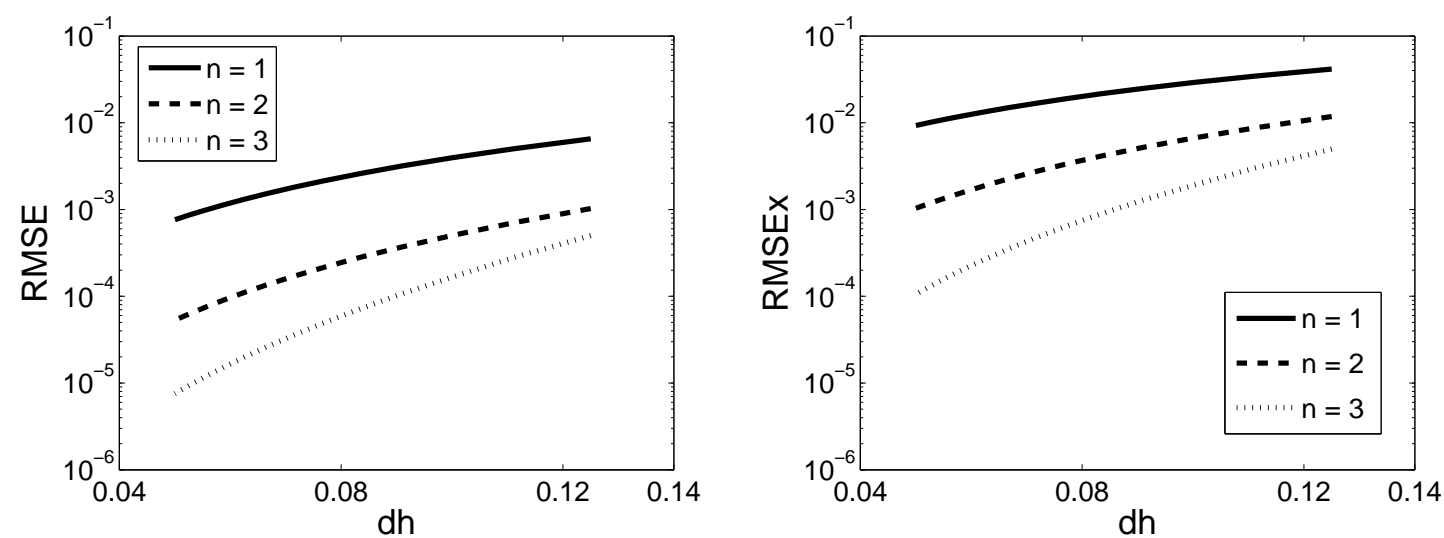

Figure 7: RMSE (left) and RMSEx (right) obtained by different order of conical RBF, $r^{2 m-1}$, for the Dirichlet-boundary-condition problem.
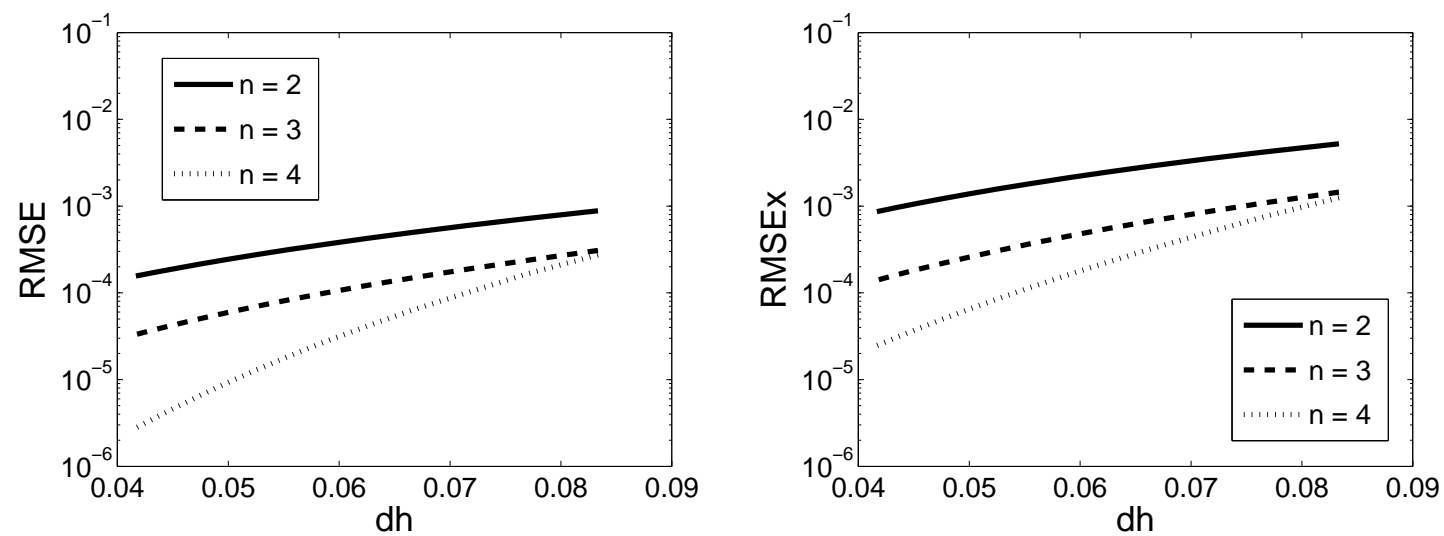

Figure 8: RMSE (left) and RMSEx (right) obtained by different order of conical RBF, $r^{2 m-1}$, for mixed-boundary-condition problem. 
extended to solving more challenging problems. Similar to Kansa's method, our proposed method is very simple and direct. The proposed method can be easily applied to timedependent problems and nonlinear problems. Since the global RBFs are used in the solution process, the ill-conditioning of the resultant matrix is an issue for solving largescaled problems. Our next goal is to investigate our current method using local RBFs so that we can solve large-scaled problems in science and engineering.

Acknowledgement: The first author acknowledges the support of NATO Collaborative Linkage Grant under reference ESP.CLG.982891.

\section{References}

[1] K.E. Atkinson. The numerical evaluation of particular solutions for Poisson's equation. IMA Journal of Numerical Analysis, 5:319-338, 1985.

[2] C.S. Chen, C.M. Fan, and J. Monroe. The method of fundamental solutions for solving elliptic partial differential equations with variable coefficients. In Y.S. Smyrlis C.S. Chen, A. Karageorghis, editor, The Method of Fundamental Solutions - A Meshless Method, pages 75-105. Dynamics Publisher, 2008.

[3] C.S. Chen, C.M. Fan, and P.H. Wen. The method of particular solutions for solving certain partial differential equations. submitted.

[4] A.H.-D. Cheng. Particular solutions of laplacian, helmholtz-type, and polyharmonic operators involving higher order radial basis functions. Eng. Analy. Boundary Elements, 24:531-538, 2000.

[5] H.A. Cho, M.A. Golberg, A.S. Muleshkov, and X. Li. Trefftz methods for time dependent partial differential equations. Computers, Materials, and Continua, 1:138,2004 .

[6] G Fairweather and A. Karageorghis. The method of fundamental solution for elliptic boundary value problems. Advances in Computatonal Mathematics, 9:69-95, 1998.

[7] L. Fox, P. Henrici, and C.B. Moler. Approximations and bounds for eigenvalues of elliptic operators. SIAM J. Numer. Anal., 4:89-102, 1967.

[8] M.A. Golberg and C.S. Chen. The method of fundamental solutions for potential, Helmholtz and diffusion problems. In M.A. Golberg, editor, Boundary Integral Methods: Numerical and Mathematical Aspects, pages 103-176. WIT Press, 1998.

[9] E.J. Kansa. Multiquadrics - a scattered data approximation scheme with applications to computational fluid dynamics - I. Comput. Math. Applic., 19(8/9):127-145, 1990 . 
[10] V.D. Kupradze and M.A. Aleksidze. The method of functional equations for the approximate solution of certain boundary value problems. U.S.S.R. Computational Mathematics and Mathematical Physics, 4:82-126, 1964.

[11] P.W. Partridge, C.A. Brebbia, and L.C. Wrobel. The Dual Reciprocity Boundary Element Method. CMP/Elsevier, 1992.

[12] S.Y. Reutskiy, C.S. Chen, and H.Y. Tian. A boundary meshless method using chebyshev interpolation and trigonometric basis function for solving heat conduction problems. International Journal Numerical Methods in Engineering, 74:1621-1644, 2008 .

[13] C.C. Tsai, A. H-D. Cheng, and C.S. Chen. Particular solutions of splines and monomials for polyharmonic and products of helmholtz operators. Engineering Analysis with Boundary Elements, to appear.

[14] H. Wang and Q.H. Qin. A meshless method for generalized linear or nonlinear poisson-type problems. Engineering Analysis with Boundary Elements, 30:515-521, 2006. 